\title{
An elusive mimic of sacroiliitis: diagnostic dilemma for the rheumatologist
}

\author{
Jacopo Ciaffi ${ }^{1}$ (D) Elena Borlandelli ${ }^{2} \cdot$ Cecilia Tetta $^{3} \cdot$ Marco Miceli $^{3} \cdot$ Riccardo Meliconi $^{1,4} \cdot$ Francesco Ursini $^{1,4}$
}

Received: 14 February 2021 / Revised: 12 March 2021 / Accepted: 22 March 2021 / Published online: 5 April 2021

(C) International League of Associations for Rheumatology (ILAR) 2021

\section{Case presentation}

A 42-year-old woman was evaluated in the orthopedic clinic because of persistent dull pain, worse at night, in the lower back and left buttock. At physical examination, FABER test (Patrick's test) and sacroiliac compression test were positive on the left side, while straight leg raise test (Lasègue's sign) was negative. Laboratory tests were unremarkable, with erythrocyte sedimentation rate and $\mathrm{C}$-reactive protein within normal limits. HLA-B27 was absent. MRI revealed a left iliac bone area of signal hyperintensity in STIR sequences (Fig. 1, panel A). Two years before current presentation, the patient had been treated with endoscopic resection for recurrent low-grade urothelial carcinoma, raising the possibility of a bone metastasis. FDG PET/CT was obtained and the lesion had a SUV of 6.4. No other areas of abnormal FDG uptake were detected. A CT-guided percutaneous needle biopsy showed non-specific eosinophilic inflammatory infiltrate and no evidence of neoplastic disease. After 3 months, repeat MRI revealed an increase of the known lesion and a new area with similar characteristics in the contralateral sacral bone (Fig. 1, panel B). The case was then re-evaluated in a multidisciplinary discussion with two rheumatologists. A differential diagnosis of active sacroiliitis in axial spondyloarthritis was

Jacopo Ciaffi

jacopo.ciaffi91@gmail.com

1 Medicine \& Rheumatology Unit, IRCCS Istituto Ortopedico Rizzoli, Bologna, Italy

2 Radiology Unit, Department of Experimental, Diagnostic and Specialty Medicine, S. Orsola Malpighi University Hospital, Bologna, Italy

3 Diagnostic and Interventional Radiology Unit, IRCCS Istituto Ortopedico Rizzoli, Bologna, Italy

4 Department of Biomedical and Neuromotor sciences (DIBINEM), University of Bologna, Bologna, Italy considered likely and treatment with celecoxib $200 \mathrm{mg}$ daily was initiated. However, after 3 months, the patient reported no response to treatment and, due to persisting concerns of malignancy, a third biopsy was performed, disclosing the real nature of the elusive lesion. An indolent B cell lymphoma was finally diagnosed and the patient was referred to the hematology unit. At the time of writing, radiation therapy was initiated to treat the left iliac bone lesion while close follow-up was decided for the right sacral lesion.

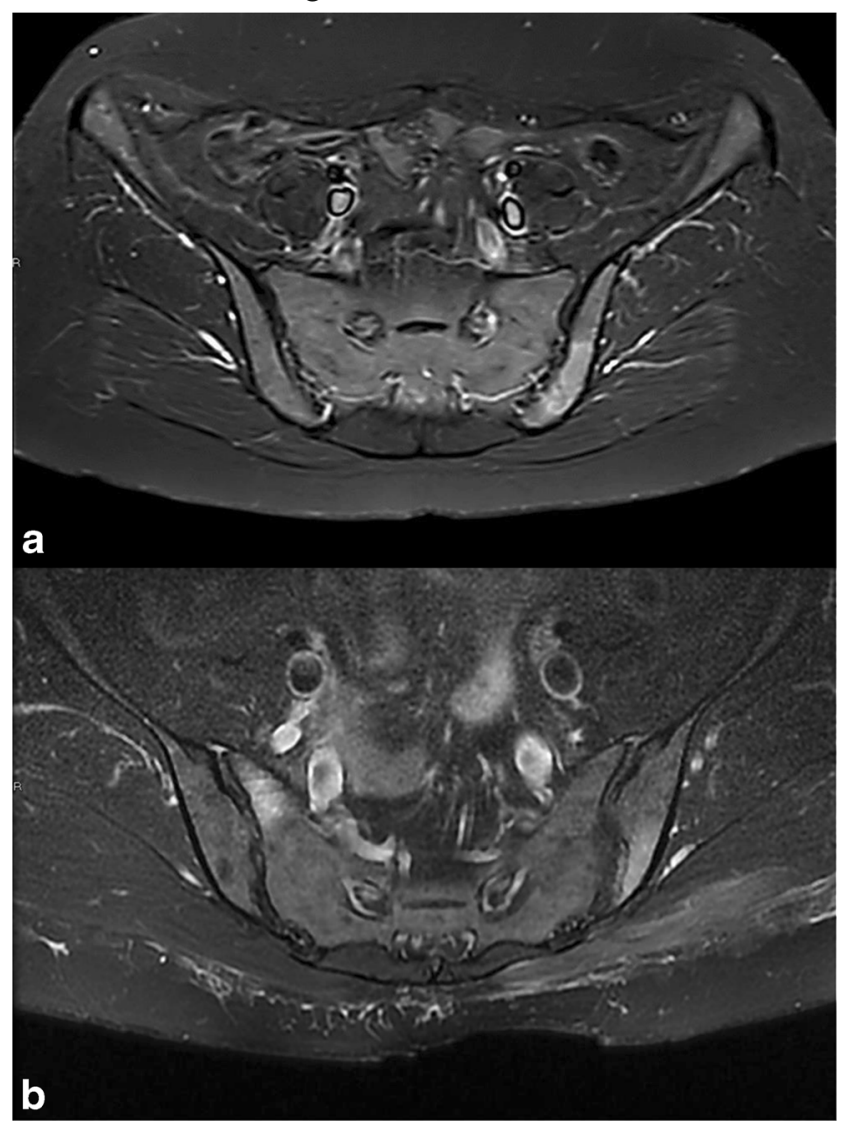

Fig. 1 Panel a: axial magnetic resonance imaging (MRI) short-tau inversion recovery (STIR) sequence showing signal hyperintensity in the left iliac bone. Panel b: axial STIR sequence showing signal hyperintensity in the left iliac bone and right sacral bone 


\section{Discussion}

The presence of active sacroiliitis [1] is pivotal to diagnose axial spondyloarthritis [2]. However, different mimics of sacroiliitis have been described. Paget disease, diffuse idiopathic skeletal hyperostosis, sarcoidosis, infections [3], and also bone and hematologic tumors [4-6] might present with radiologic features suggestive of sacroiliitis. As highlighted by our report, finding the correct diagnosis may be challenging in these cases. Rheumatologists should keep a high level of suspicion for malignancy in particular in patients with a prior history of cancer, with atypical presentation of sacroiliitis, or with unrelieved symptoms despite nonsteroidal anti-inflammatory drugs.

\section{Declarations}

Patient consent Patient's written informed consent for publication was obtained.

Disclosures None

\section{References}

1. Lambert RGW, Bakker PAC, van der Heijde D, Weber U, Rudwaleit M, Hermann K-GA, Sieper J, Baraliakos X, Bennett A, Braun J, Burgos-Vargas R, Dougados M, Pedersen SJ, Jurik AG, Maksymowych WP, Marzo-Ortega H, Østergaard M, Poddubnyy D, Reijnierse M, van den Bosch F, van der Horst-Bruinsma I, Landewé R (2016) Defining active sacroiliitis on mri for classification of axial spondyloarthritis: Update by the asas mri working group. Ann Rheum Dis 75:1958-1963

2. Rudwaleit M, Van Der Heijde D, Landewe R, Listing J, Akkoc N, Brandt $\mathrm{J}$ et al (2009) The development of assessment of spondyloarthritis international society classification criteria for axial spondyloarthritis (part ii): Validation and final selection. Ann Rheum Dis 68:777-783

3. Antonelli MJ, Magrey M (2017) Sacroiliitis mimics: a case report and review of the literature. BMC Musculoskelet Disord 18:170

4. Solmaz D, Onen F, Balci A, Akar S (2013) Pelvic ewing sarcoma mimicking sacroiliitis. Arthritis Rheum 65:290

5. Moghadam A, Talebi-Taher M, Dehghan A (2010) Sacroiliitis as an initial presentation of acute lymphoblastic leukaemia. Acta Clin Belg 65:197-199

6. Duman I, Aydemir K, Tan AK, Dinçer K, Kalyon TA (2007) An unusual case of osteoid osteoma clinically mimicking sacroiliitis. Clin Rheumatol 26:1158-1160

Publisher's note Springer Nature remains neutral with regard to jurisdictional claims in published maps and institutional affiliations. 